\title{
GYY4137, a hydrogen sulfide-releasing molecule, inhibits the inflammatory response by suppressing the activation of nuclear factor-kappa $B$ and mitogen-activated protein kinases in Coxsackie virus B3-infected rat cardiomyocytes
}

\author{
ZUBO WU, HUA PENG, QING DU, WEN LIN and YALI LIU \\ Department of Pediatrics, Union Hospital, Tongji Medical College, Huazhong University of Science and Technology, \\ Wuhan, Hubei 430022, P.R. China
}

Received December 1, 2013; Accepted July 21, 2014

DOI: $10.3892 / \mathrm{mmr} .2014 .2901$

\begin{abstract}
GYY4137 is a water-soluble, small molecule hydrogen sulfide $\left(\mathrm{H}_{2} \mathrm{~S}\right)$-release agent that possesses potent cardioprotective and anti-inflammatory properties in experimental models. Coxsackie virus B3 (CVB3) infection commonly causes viral myocarditis, which mainly involves immune cell infiltration, eventually resulting in heart failure. In the present study, the effects and underlying mechanisms of GYY4137 treatment of CVB3-induced myocarditis were investigated. The effects of GYY4137 on CVB3-induced nuclear factor-kappa $\mathrm{B}(\mathrm{NF}-\kappa \mathrm{B})$ activity were examined by western blotting, immunofluorescence and electrophoretic mobility shift assay. Mitogen-activated protein kinase (MAPK) signaling protein expression levels were detected by western blotting. Cardiomyocyte damage-related enzyme activities, such as lactate dehydrogenase (LDH) and creatine kinase MB (CK-MB), were measured by ELISA, as well as the production of proinflammatory cytokines. The results revealed that GYY4137 suppressed CVB3-induced secretion of LDH, CK-MB and pro-inflammatory cytokines, such as tumor necrosis factor- $\alpha$, interleukin (IL)-1 $\beta$ and IL-6. Furthermore, the activation of $\mathrm{NF}-\kappa \mathrm{B}$ and the $\mathrm{I} \kappa \mathrm{B} \alpha$ degradation induced by CVB3 were also inhibited by GYY4137. Notably, the phosphorylation of p38, ERK1/2 and JNK1/2 induced by CVB3 was also inhibited by GYY4137. In conclusion, the data demonstrate that GYY4137 exerts anti-inflammatory effects in CVB3-infected cardiomyocytes. This anti-inflammatory mechanism may be associated with suppression of NF- $\mathrm{NB}$ and MAPK signaling pathway activation.
\end{abstract}

Correspondence to: Professor Yali Liu, Department of Pediatrics, Union Hospital, Tongji Medical College, Huazhong University of Science and Technology, 1277 Liberation Avenue, Wuhan, Hubei 430022, P.R. China

E-mail:whlyl1023@163.com

Key words: GYY4137, coxsackie virus B3, cardiomyocytes, nuclear factor- $\mathrm{\kappa} \mathrm{B}$, mitogen activated protein kinase

\section{Introduction}

Coxsackie virus B3 (CVB3) infection is considered to be a predominant cause of human myocarditis, and infection may eventually result in heart failure (1). CVB3-induced myocarditis is mainly caused by inflammatory infiltration of myocardial tissue, and remodeling of cardiac structure and function (2). Although the virus directly attacks myocardial cells, activation of the immune response and a subsequent inflammatory cascade is key to the pathogenesis of CVB3-mediated viral myocarditis (VM). In a previous model of CVB3 infection, nuclear factor $(\mathrm{NF})-\kappa \mathrm{B}$ was shown to be vital in regulating host inflammatory defenses (3). NF- $\kappa \mathrm{B}$ mediates the expression of various pro-inflammatory cytokines, such as tumor necrosis factor (TNF)- $\alpha$, interleukin (IL)-1 $\beta$ and IL-6, and thus may aggravate myocardial injury (4). Notably, NF- $\mathrm{B}$ inhibitors, including pyrrolidine dithiocarbamate and Bay 11-7082 were demonstrated to significantly inhibit the myocardial tissue expression of pro-inflammatory factors, slowing myocarditis pathogenesis (5). Despite extensive investigation into VM prevention and treatment, there remains no definitive therapy for this disease.

Hydrogen sulfide $\left(\mathrm{H}_{2} \mathrm{~S}\right)$ is generated in mammalian tissues mainly by two types of pyridoxal-5'-phosphate-dependent enzymes, cystathionine $\beta$-synthase and cystathionine $\gamma$-lyase (6). $\mathrm{H}_{2} \mathrm{~S}$ has long been recognized as a toxic gas with a pungent odor, which pollutes the environment and endangers human health. However, a previous study has shown that $\mathrm{H}_{2} \mathrm{~S}$ may become the third endogenous gaseous signaling transmitter identified, following nitric oxide (NO) and carbon monoxide, due to newly-detected physiological and pathophysiological functions (7). It has been demonstrated that $\mathrm{H}_{2} \mathrm{~S}$ exerts protective effects against myocardial ischemia (8); however, great controversy remains regarding the exact role of $\mathrm{H}_{2} \mathrm{~S}$ in inflammation (9). Furthermore, considerable evidence suggests that $\mathrm{H}_{2} \mathrm{~S}$ also exerts an anti-apoptotic effect $(10,11)$. In local and systemic inflammation experimental models, $\mathrm{H}_{2} \mathrm{~S}$ was shown to have a pro-inflammatory role (12). Conversely, accumulating evidence suggests that $\mathrm{H}_{2} \mathrm{~S}$ exerts an anti-inflammatory effect in various models $(13,14)$. In endotoxic shock rat models, $\mathrm{H}_{2} \mathrm{~S}$ inhibited lipopolysaccharide (LPS)-mediated inflammatory cytokine production and reduced plasma C-reactive protein (15). 
In LPS-irritated human cartilage cells, the $\mathrm{H}_{2} \mathrm{~S}$ release agent GYY4137 significantly repressed cyclooxygenase 2, inducible $\mathrm{NO}$ synthase and TNF- $\alpha$ expression, by restraining the activation of NF- $\kappa \mathrm{B}$ (16). To confirm the potent anti-inflammatory mechanism of $\mathrm{H}_{2} \mathrm{~S}$ in $\mathrm{CVB} 3$-infected cardiomyocytes, the present study investigated whether $\mathrm{H}_{2} \mathrm{~S}$, administered via GYY4137 treatment, exerts anti-inflammatory effects in CVB3-infected cardiomyocytes, and whether this occurs through the inhibition of pro-inflammatory processes, such as the downregulation of IL-6, IL-1 $\beta$ and TNF- $\alpha$.

Mitogen-activated protein kinases (MAPKs) are considered to constitute an intracellular signal transduction pathway important in inflammatory reactions induced by viral infections and oxidative stress, and are involved in regulating the expression levels of various inflammatory factors and cell adhesion factors (17). In cell ligation and puncture-induced models of sepsis, $\mathrm{H}_{2} \mathrm{~S}$ was demonstrated to regulate the systemic inflammatory response via the MAPK signaling pathway (18). In a mouse model of VM, the MAPK signaling pathway (ERK1/2, p38 and JNK1/2) was shown to be activated and involved in the inflammatory reaction, upregulating inflammatory factor expression (19). In the present study, the underlying mechanism and intracellular signaling pathways affected by $\mathrm{H}_{2} \mathrm{~S}$ in CVB3-infected cardiomyocytes were investigated.

\section{Materials and methods}

Animals. Sprague Dawley rats, aged 1-2-days, were purchased from the Center of Experimental Animals of Tongji Medical College, Huazhong University of Science and Technology, Wuhan, China. All animals used in this study were cared for in accordance with the Guide for the Care and Use of Laboratory Animals published by the United States National Institute of Health (NIH publication no. 85-23, revised 1996) and all procedures were approved by the committee of Experimental Animals of Tongji Medical College, Huazhong University of Science and Technology.

Reagents. Dulbecco's modified Eagle's medium (DMEM)/F12, Trypsin $0.25 \%$ EDTA and fetal bovine serum (FBS) were obtained from Gibco-BRL (Carlsbad, CA, USA). Collagenase B was purchased from Roche Diagnostics $\mathrm{GmbH}$ (Mannheim, Germany). GYY4137 was bought from Cayman Chemical Company (Ann Arbor, MI, USA). The IL-6, IL-1 $\beta$ and TNF- $\alpha$ ELISA kits were purchased from USCN Life Science, Inc. (Wuhan, China). The Cell Counting kit-8 (CCK-8) was obtained from Dojindo Molecular Technologies (Kunamoto, Japan). 5-bromo-2'-deoxyuridine (BrdU) was purchased from Sigma-Aldrich (St. Louis, MO, USA). The lactate dehydrogenase $(\mathrm{LDH})$ and creatine kinase-MB (CK-MB) ELISA kits were bought from Nanjing Jiancheng Biochemical Reagent Co., Ltd (Nanjing, China). The primary antibodies for the NF- $\kappa \mathrm{B}$ and MAPK signaling pathways were from Cell Signaling Technology, Inc. (Danvers, MA, USA) and horse radish peroxidase (HRP)-conjugated secondary antibodies were purchased from Santa Cruz Biotechnology, Inc. (Santa Cruz, CA, USA).

CVB3 virus. A Nancy variant of CVB3, which was provided by the State Key Laboratory of Virology (Wuhan, China), was propagated in $\mathrm{HeLa}$ cell monolayers and stored at $-80^{\circ} \mathrm{C}$ until use. The HeLa cells were provided by Prof. ZeHua Wang (Department of Obstetrics and Gynecology, Union Hospital, Tongji Medical College, Huazhong University of Science and Technology, Wuhan, China.). The HeLa cells were grown in DMEM, supplemented with $10 \%$ FBS. After reaching $\geq 90 \%$ confluence, CVB3 was added to the cells for $2 \mathrm{~h}$, the virus was then released from the cells by three freeze-thaw cycles. The samples were centrifuged at $5,000 \mathrm{x} \mathrm{g}$ at $4^{\circ} \mathrm{C}$ for $10 \mathrm{~min}$, and the supernatants were collected. The viral titer was determined to be $10^{-4.5}$ in terms of the viral cytopathic effects, and was expressed as the tissue culture infective dose $\left(\mathrm{TCID}_{50}\right)$.

Primary culture of neonatal rat cardiomyocytes. The 1-2-days old Sprague Dawley rats were sacrificed by ether inhalation and the hearts were harvested. The primary cardiomyocytes were isolated from the ventricles, as described previously (20), with certain modifications. Briefly, the hearts were lightly minced and digested with $0.05 \%$ trypsin and $0.025 \%$ collagenase $\mathrm{B}$ at $37^{\circ} \mathrm{C}$ for $5 \mathrm{~min}$. Subsequent to digestion five times, the supernatants were filtered, centrifuged for $10 \mathrm{~min}\left(120 \mathrm{x} \mathrm{g}, 4^{\circ} \mathrm{C}\right)$ and resuspended in the complete medium, which consisted of DMEM/F12 containing $10 \% \mathrm{FBS}, 100 \mathrm{U} / \mathrm{ml}$ penicillin and $100 \mathrm{ug} / \mathrm{ml}$ streptomycin. The resuspended cells were then plated in a petri dish in a humidified incubator $\left(5 \% \mathrm{CO}_{2}, 37^{\circ} \mathrm{C}\right)$ for $1.5 \mathrm{~h}$ to reduce the numbers of endothelial cells and cardiac fibroblasts. Any non-adherent cells were collected, filtered once, resuspended in the complete medium and seeded in 6-well plates. After 48 h, myocyte morphology was observed and the cells started beating spontaneously. By observation of the cell morphology and beat frequency, $>90 \%$ cells were identified as cardiomyocytes. The cells were incubated for three days prior to further experiments.

Cell treatment. Prior to each experiment, the cardiomyocytes were serum-starved for $12 \mathrm{~h}$. The cells were then treated with different concentrations of GYY4137 $(10,50$ and $100 \mu \mathrm{M})$ for different time periods prior to the addition of $100 \mathrm{TCID}_{50}$ CVB3 for $2 \mathrm{~h}$ under serum-free conditions.

Cell viability assay. The CCK-8 assay was used to detect cell activity. The cardiomyocytes were seeded at a density of $1 \times 10^{4}$ cells/well in a 96 -well plate in order to observe the potential cytotoxicity. The cells were serum-starved for $12 \mathrm{~h}$ and then incubated with GYY4137 $(10-100 \mu \mathrm{M})$ for a further $24 \mathrm{~h}$. Following the indicated treatments, $10 \mu \mathrm{l} \mathrm{CCK}-8$ solution was added to each well of the plate and the cells were incubated for an additional $4 \mathrm{~h}$ at $37^{\circ} \mathrm{C}$. The absorbance at $450 \mathrm{~nm}$ was measured with a microplate reader (Bio-Rad, Hercules, CA, USA). The mean optical density (OD) of six wells in the indicated groups were used to calculate the percentage cell viability using the following calculation: Percentage cell viability $=\left(\mathrm{OD}_{\text {treatment group }} / \mathrm{OD}_{\text {control group }}\right) \times 100 \%$. The experiment was repeated three times.

$L D H$ and $C K-M B$ assays. The cells were pre-incubated with $100 \mathrm{TCID}_{50} \mathrm{CVB} 3$ for $2 \mathrm{~h}$ and then different concentrations of GYY4137 $(10,50$ and $100 \mu \mathrm{M})$ for another $72 \mathrm{~h}$. The supernatant media from the myocardial cell cultures were collected 
from all groups and stored at $-80^{\circ} \mathrm{C}$. $\mathrm{LDH}$ and CK-MB isoenzyme activity in all media samples was measured according to the manufacturer's instructions of the respective ELISA kits. Each measurement was repeated three times. Enzyme activity was indicated by the enzyme unit (U), which signified the quantity of a particular enzyme.

Cytokine assays. The cardiomyocytes were cultured in 96-well plates $\left(1 \times 10^{4}\right.$ cells $\left./ \mathrm{ml}\right)$ and preincubated with 100 TCID $_{50}$ CVB3 for $2 \mathrm{~h}$, followed by incubation with different concentrations of GYY4137 $(10,50$ and $100 \mu \mathrm{M})$ for another $24 \mathrm{~h}$. The cell culture supernatants were used to measure the IL-6, IL-1 $\beta$ and TNF- $\alpha$ levels using ELISA kits, following the manufacturer's instructions.

Electrophoretic mobility shift assay (EMSA). The NF- $\mathrm{B}$ DNA-banding activity was detected by EMSA using a commercial kit (Thermo Fisher Scientific, Rockford, IL, USA). The cardiomyocytes were plated in $100 \mathrm{~mm}$ dishes $\left(1 \times 10^{6}\right.$ cells per dish) and grown in complete DMEM/F12 until confluent. The cells were preincubated with 100 TCID $_{50}$ CVB3 for $2 \mathrm{~h}$ and then incubated with different concentrations of GYY4137 (10, 50 and $100 \mu \mathrm{M}$ ) for another $12 \mathrm{~h}$. Subsequent to washing three times with cold phosphate-buffered saline (PBS; pH 7.4), the cells were then scraped and centrifuged at 15,000 x g for $5 \mathrm{~min}$, to collect the pellets. Nuclear proteins were extracted using the NE-PER ${ }^{\circledR}$ Nuclear Extraction Reagent (Thermo Fisher Scientific) according to the manufacturer's instructions. Biotin-labeled $\mathrm{NF}-\kappa \mathrm{B}$ specific oligonucleotides, with the following sequence: 5'-AGTTGAGGGGACTTTCCCAGGC-'3, were prepared as labeled probes, according to the manufacturer's instructions (Promega Corporation, Madison, WI, USA). Biotin end-labeled double-stranded DNA and the nuclear extracts were incubated at room temperature for $20 \mathrm{~min}$, and then $10 \mu \mathrm{l}$ protein-DNA complex was subjected to $6.5 \%$ PAGE at $100 \mathrm{~V}$ for $1 \mathrm{~h}$ at $4^{\circ} \mathrm{C}$ and transferred to a nylon membrane. Following ultra-violet light cross-linking, HRP-labeled streptavidin incubation with chemiluminescent substrate (North2South ${ }^{\circledR}$ Chemiluminescent Hybridization and Detection kit; Thermo Fisher Scientific, Waltham, MA, USA) was used to detect the biotin end-labeled DNA. The nylon membrane was subjected to X-ray film exposure and the results obtained were analyzed using AlphaEase software (Alpha Innotech, Santa Cruz, CA, USA).

Western blot analysis of protein expression levels. Subsequent to infection with CVB3 for $2 \mathrm{~h}$, the cells were treated with GYY4137 at concentrations of 10,50 and $100 \mu \mathrm{M}$ for different time periods ( $6 \mathrm{~h}$ for detection of the MAPK signaling pathway, $24 \mathrm{~h}$ for the $\mathrm{NF}-\kappa \mathrm{B}$ signaling pathway). Following the indicated treatments, the cells were harvested and lysed with cell lysis buffer (Thermo Fisher Scientific), and centrifuged at $10,000 \mathrm{x} \mathrm{g}$ for $10 \mathrm{~min}$. The supernatant served to represent total protein. Nuclear and membranous proteins were extracted using the NE-PER extraction kit, according to the manufacturer's instructions. The protein concentrations were determined using a bicinchoninic acid protein assay kit (Beyotime, Biotech., Jiangsu, China). The protein samples (40 $\mu \mathrm{g})$ were separated by $10 \%$ SDS-PAGE and transferred to polyvinylidene difluoride membranes. The membranes were blocked with 5\% non-fat milk in Tris-buffered saline containing $0.05 \%$
Tween-20 (TBST) at room temperature for $2 \mathrm{~h}$, washed and incubated at $4^{\circ} \mathrm{C}$ overnight with various primary antibodies (phosphor-ERK1/2, 1:500; ERK1/2, 1:1,000; p38, 1:1,000; JNK, 1:1,000; IкB $\alpha, \mathrm{NF}-\kappa \mathrm{B}$ p65, Histone 3 and $\beta$-actin, $1: 1,000)$. Following washing with TBST, the membranes were incubated with the HRP-conjugated secondary antibodies (goat anti-rabbit $\operatorname{IgG}$, rabbit anti-goat $\operatorname{IgG}$, rabbit anti-rat IgG and rabbit anti-mouse $\mathrm{IgG}$; all 1:3,000). Subsequent to washing the membranes, an Enhanced Chemiluminescence kit (Thermo Fisher Scientific) was used to detect the immunoreactivity, then the membranes were exposed to X-ray film. The density of the signals was quantified using AlphaEase FC software (Genetic Technologies, Inc., Miami, Florida, USA).

Immunofluorescence analysis. The cardiomyocytes were grown on glass slides in 6-well plates. Following incubation with CVB3 for $2 \mathrm{~h}$, the cells were treated with $50 \mu \mathrm{M}$ GYY4137 for a further $24 \mathrm{~h}$. The cells were washed three times and fixed with $4 \%$ paraformaldehyde for $15 \mathrm{~min}$, and then incubated with an immune blocking solution for $1 \mathrm{~h}$ to reduce non-specific binding. Subsequently, the cells were washed three times and incubated at $4^{\circ} \mathrm{C}$ overnight with the primary $\mathrm{NF}-\kappa \mathrm{B}$ p65 antibody. Following washing, the cells were incubated with Cy3-conjugated secondary antibody (1:200 dilution) at room temperature for $1 \mathrm{~h}$, then the nuclei were stained with DAPI for 5 min prior to observation. Images were captured at excitation wavelengths of $350 \mathrm{~nm}$ (for DAPI) and $540 \mathrm{~nm}$ (for Cy3). The p65 protein and DAPI-stained nuclei exhibited red and blue fluorescence, respectively, which was visualized by laser confocal microscopy (A1Si; Nikon Corporation, Tokyo, Japan). Image-Pro Plus software (Media Cybernetics, Georgia, MD, USA) was used to analyze the results.

Statistics. All experiments were repeated at least three times with equivalent results. The data are expressed as the means \pm standard error. One-way analysis of variance was used to analyze the differences between groups. The data was analyzed using SPSS 18.0 software (SPSS, Inc., Chicago, IL, USA). A $\mathrm{P}<0.05$ was considered to indicate a statistically significant difference.

\section{Results}

GYY4137 is not cytotoxic to cardiomyocytes. Following exposure of the cardiomyocytes to different GYY4137 concentrations $(10,50$ and $100 \mu \mathrm{M})$ for $24 \mathrm{~h}$, no significant differences in cell viability were detected (Fig. 1). These data indicate that different GYY4137 concentrations exerted no cytotoxic effect in cardiomyocytes; therefore, the GYY4137 concentrations used in subsequent experiments did not exceed $100 \mu \mathrm{M}$.

Cardiomyocyte damage-related enzyme activity in the supernatant of myocardial cells. The levels of LDH and $\mathrm{CK}-\mathrm{MB}$ in the supernatant media are shown in Fig. 2. In the CVB3 infection group, the levels of LDH and CK-MB were significantly increased $(\mathrm{P}<0.05$ respectively) in comparison with those of the control group. The GYY4137 treatment (at concentrations of 10, 50 and $100 \mu \mathrm{M}$ ) groups exhibited significantly reduced $\mathrm{LDH}$ and CK-MB enzyme levels 


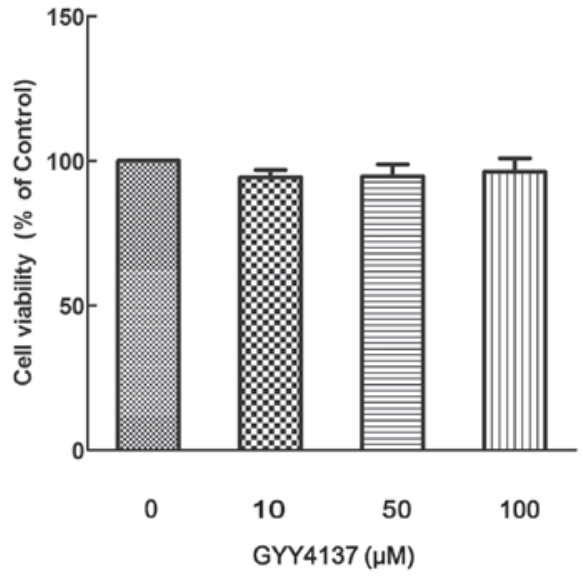

Figure 1. Analysis of cell viability upon exposure to GYY4137. GYY4137 was not observed to be cytotoxic to cardiomyocytes. The cells were treated with different concentrations $(0-100 \mu \mathrm{M})$ GYY4137 for $24 \mathrm{~h}$ and the results are expressed as the percentage of surviving cells compared to the normal cells. The cell viability was determined by cell counting kit- 8 assay. The data are expressed as the means \pm standard error of the mean from three separate experiments.

A

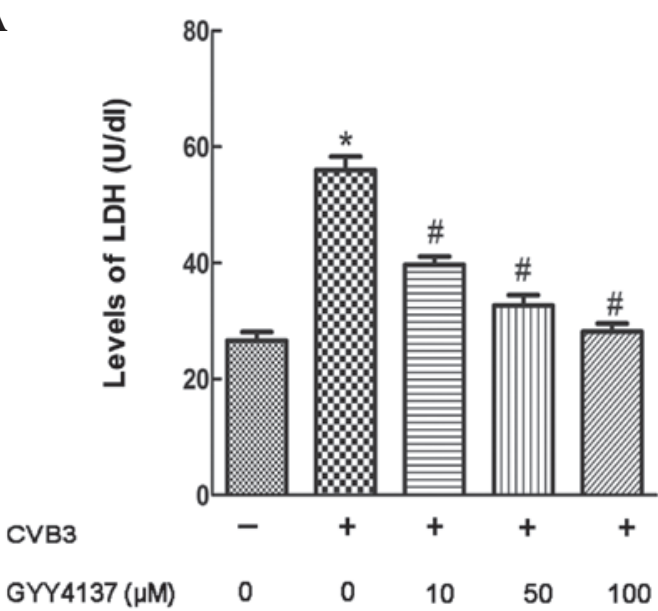

B

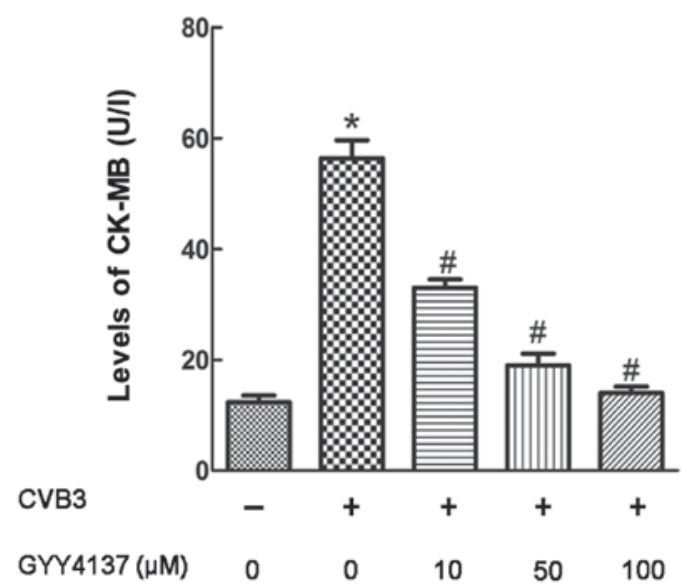

Figure 2. Cardiomyocyte damage-related enzyme activity in the supernatant of myocardial cells. Following infection with Coxsackie virus B3 (CVB3) for $2 \mathrm{~h}$, the cells were treated with GYY4137 at concentrations of $10,50,100 \mu \mathrm{M}$ for another $72 \mathrm{~h}$, with untreated cells as a control group. (A) Lactate dehydrogenase $(\mathrm{LDH})$ and $(\mathrm{B})$ creatine kinase $\mathrm{MB}(\mathrm{CK}-\mathrm{MB})$ enzyme levels were detected with commercial kits. The data are expressed as the means \pm standard error of the mean from three separate experiments. ${ }^{*} \mathrm{P}<0.05$ vs. normal control group; ${ }^{\#} \mathrm{P}<0.05$ vs. $\mathrm{CVB} 3$ group.
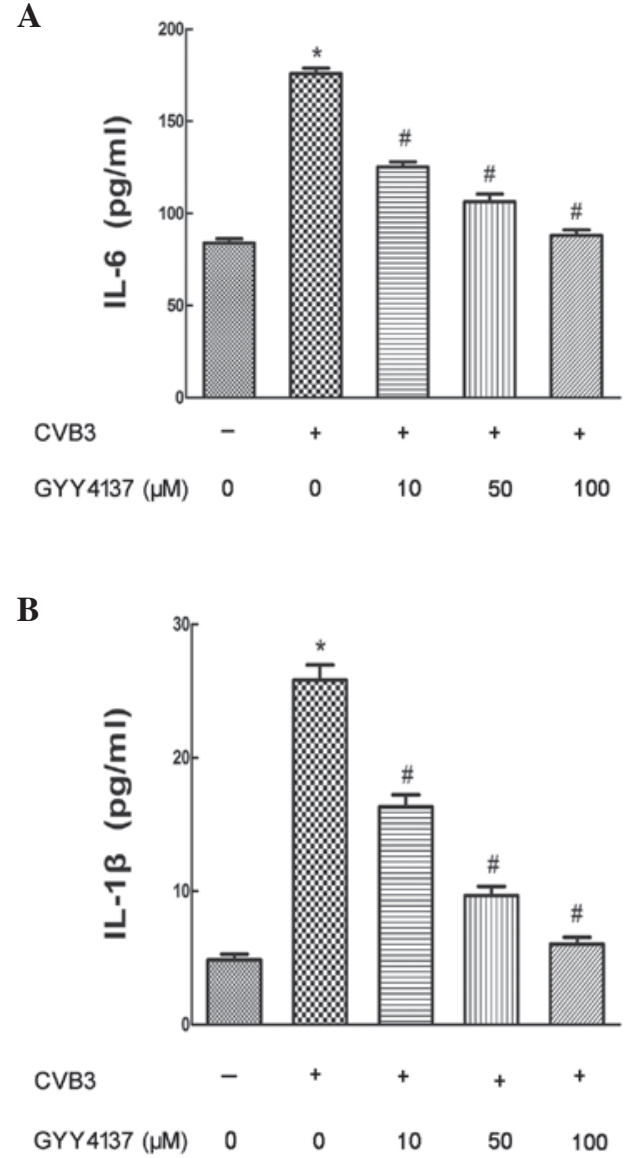

C

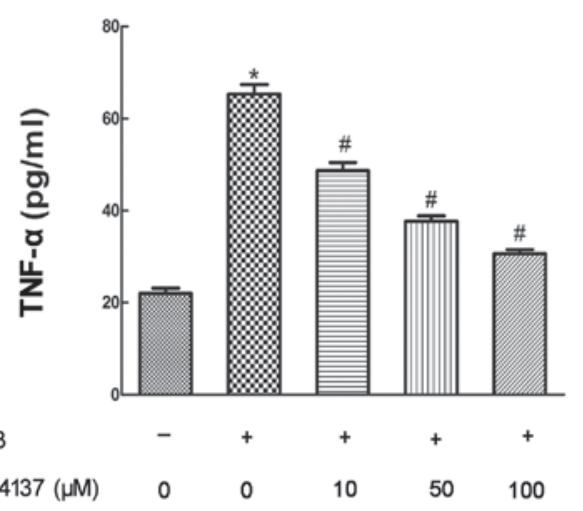

Figure 3. GYY4137 inhibits Coxsackie virus B3 (CVB3)-induced inflammatory factor secretions from cardiomyocytes. Following incubation with CVB3 for $2 \mathrm{~h}$, the cells were treated with 10, 50 and $100 \mu \mathrm{M}$ GYY4137 for $24 \mathrm{~h}$. The levels of secreted (A) interleukin (IL)-6, (B) IL-1 $\beta$ and (C) tumor necrosis factor (TNF)- $\alpha$ were measured by ELISA. The data are expressed as the means \pm standard error of the mean from three separate experiments. ${ }^{*} \mathrm{P}<0.05$ vs. normal control group; ${ }^{\#} \mathrm{P}<0.05$ vs. $\mathrm{CVB} 3$ group.

( $\mathrm{P}<0.05$ respectively) in comparison with those of the CBV3 infection group. These data indicate that GYY4137 protects against damage to myocytes in viral infection.

GYY4137 inhibits CVB3-induced cardiomyocyte inflammatory factor secretion. The cardiomyocytes were incubated with CVB3 (100 TCID $_{50}$ ) with or without GYY4137 treatment $(10,50$ and $100 \mu \mathrm{M})$. The levels of inflammatory cytokines in the cell culture media were determined by ELISA assays. As shown in Fig. 3 , the production of IL-6, IL-1 $\beta$ and TNF- $\alpha$ was 
A

B

C

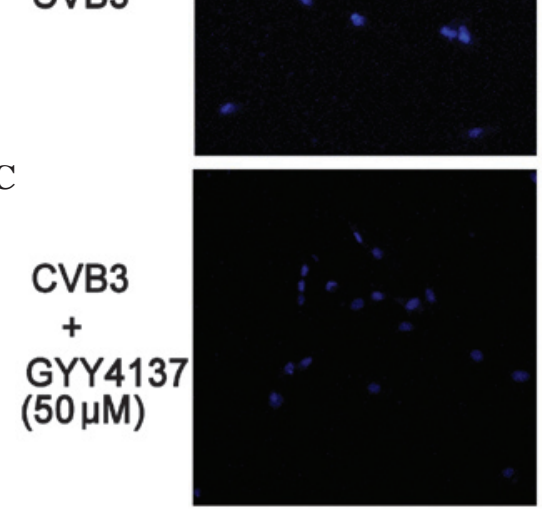

DAPI
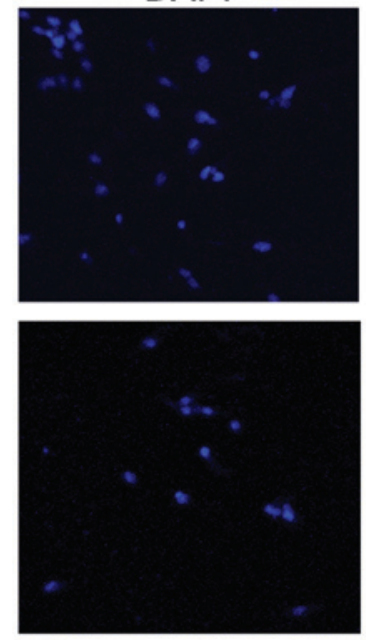

CVB3
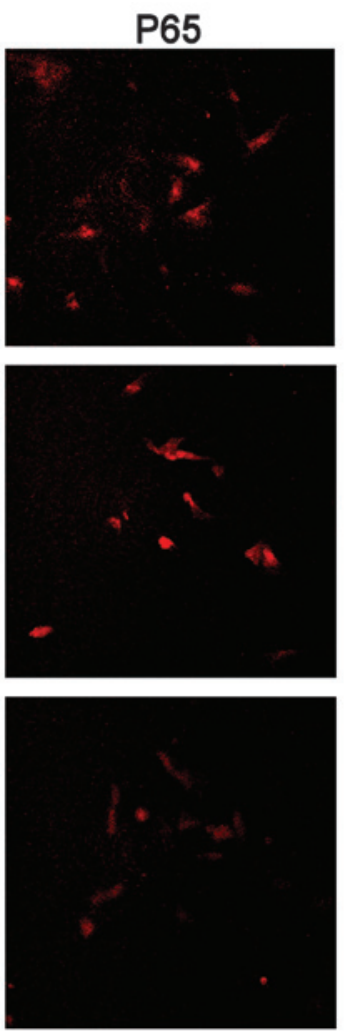
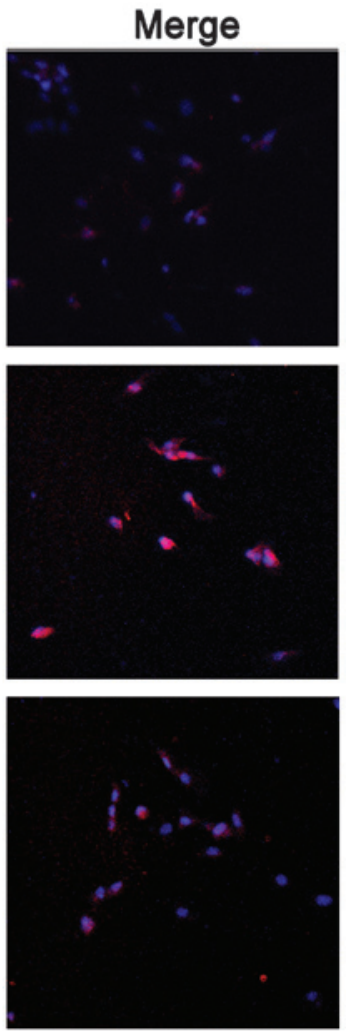

Figure 4. GYY4137 inhibits nuclear factor (NF)- $\mathrm{KB}$ translocation to the nucleus in cardiomyocytes. (A) Cells without any treatment served as a control. (B) Cells incubated with Coxsackie virus B3 (CVB3) alone. (C) Following CVB3 incubation for $2 \mathrm{~h}$, the cells were treated with $50 \mu$ M GYY4137 for another $24 \mathrm{~h}$. The images, from left to right, indicate the nuclei, p65 protein expression and merged images. The nuclei were stained by DAPI (blue), p65 was detected by Cy3-labeled immunostaining (red). The images are representative of three independent experiments.
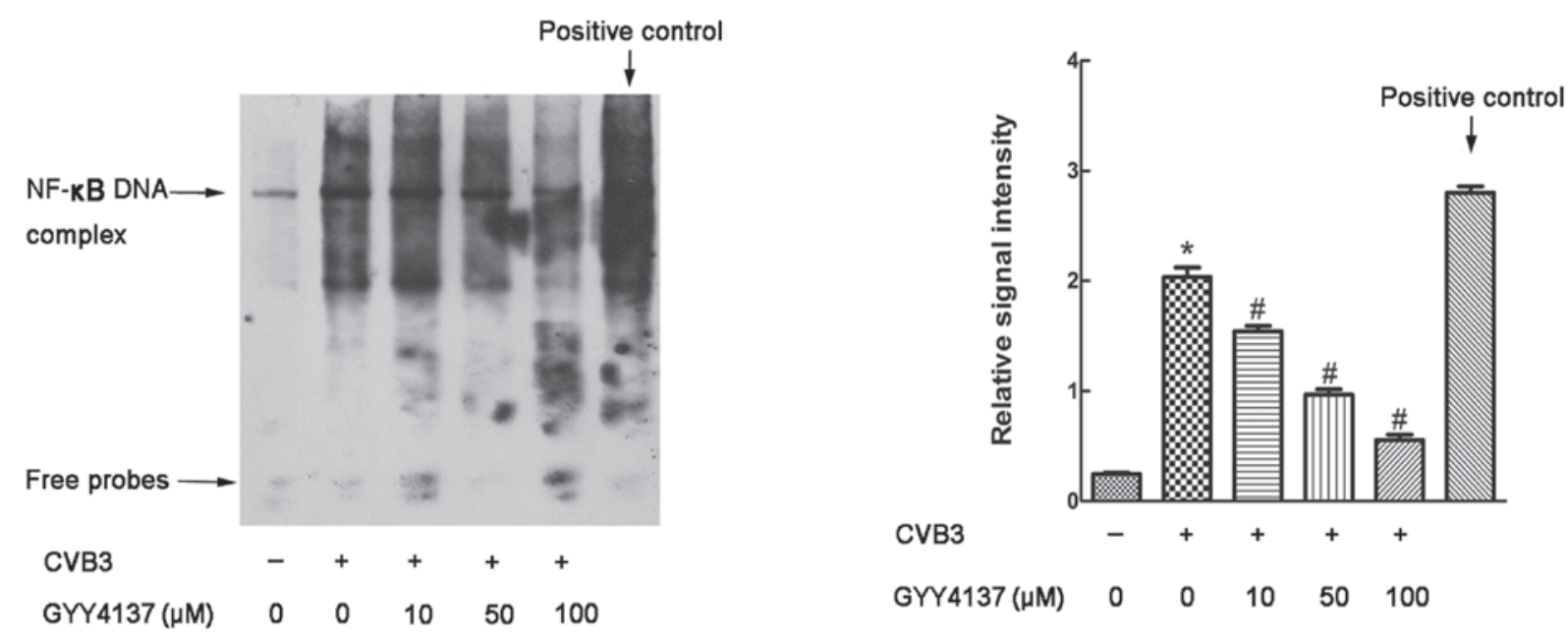

Figure 5. GYY4137 abolishes Coxsackie virus B3 (CVB3)-mediated nuclear factor (NF)-кB DNA binding activity in cardiomyocytes. Following infection with CVB3 for $2 \mathrm{~h}$, the cells were treated with GYY4137 at concentrations of 10,50 and $100 \mu \mathrm{M}$ for another $12 \mathrm{~h}$. To confirm the effect of GYY4137 on NF- $\mathrm{kB}$ activation, the binding affinity of NF- $\mathrm{KB}$ to DNA was determined by electrophoretic mobility shift assay. Extracts of lipopolysaccharide-induced RAW264.7 cells served as a positive control. The data are expressed as the means \pm standard error of the mean from three separate experiments. "P<0.05 vs. normal control group; ${ }^{*} \mathrm{P}<0.05$ vs. CVB3 group.

significantly increased following infection with CVB3, as compared with the unstimulated control $(\mathrm{P}<0.05)$. Incubating the cells with GYY4137 at 10,50 and $100 \mu \mathrm{M}$ concentrations dose-dependently inhibited the production of IL-6, IL-1 $\beta$ and TNF- $\alpha$, respectively (all $\mathrm{P}<0.05$ ). These results indicate that GYY4137 effectively inhibited the expression of inflammatory cytokines in CVB3-infected myocardial cells.
GYY4137 inhibits NF- $\kappa B$ translocation to the nucleus. To assess the potential effects on NF- $\kappa B$ translocation, indirect immunofluorescence techniques were used to detect the distribution of NF- $\kappa \mathrm{B}$ in the myocardial cells. As shown in Fig. 4, compared with normal cells (shown as control), intracellular p65 was observed to transfer from the cytoplasm to the nucleus in CVB3-infected cells. Subsequent to 
A
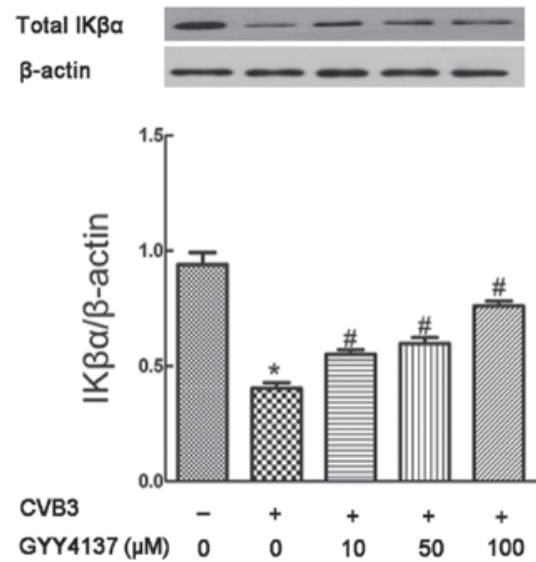

B
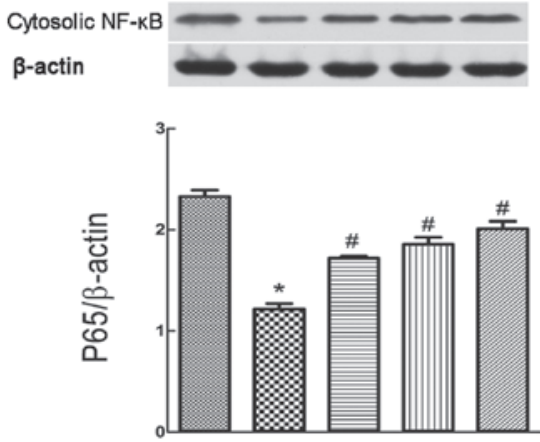

CVB3

$\operatorname{GYY4137(\mu M)} \quad 0 \quad 0 \quad 10 \quad 50 \quad 100$

C
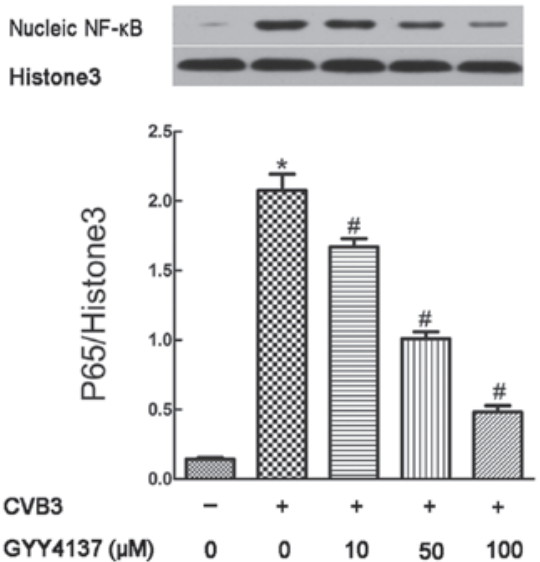

Figure 6. Effects of GYY4137 on the activation of nuclear factor (NF)- $\kappa$ B and $\mathrm{I} \kappa \mathrm{B} \alpha$ degradation in cardiomyocytes. Following infection with Coxsackie virus B3 (CVB3) for $2 \mathrm{~h}$, the cells were treated with GYY4137 at concentrations of 10,50 and $100 \mu \mathrm{M}$ for a further $24 \mathrm{~h}$, with normal cells serving as a control group. Cytosolic and nucleic protein activation of the NF- $\kappa \mathrm{B}$ signaling pathway was analyzed by western blotting. Effects of GYY4137 on (A) total $\mathrm{I} \kappa \mathrm{B} \alpha$, and (B) cytosolic and (C) nucleic NF- $\kappa \mathrm{B}$ expression levels. The $\mathrm{I} \kappa \mathrm{B} \alpha$ and cytosolic NF- $\kappa \mathrm{B}$ protein expression levels were normalized to those of $\beta$-actin. Nucleic NF- $\kappa$ B levels were normalized to those of histone 3 . The data are expressed as the means \pm standard error of the mean from three separate experiments. ${ }^{*} \mathrm{P}<0.05$ vs. normal control group; ${ }^{\#} \mathrm{P}<0.05$ vs. CVB3 group.

incubation with $50 \mu \mathrm{M}$ GYY4137, this nuclear translocation was suppressed.
A

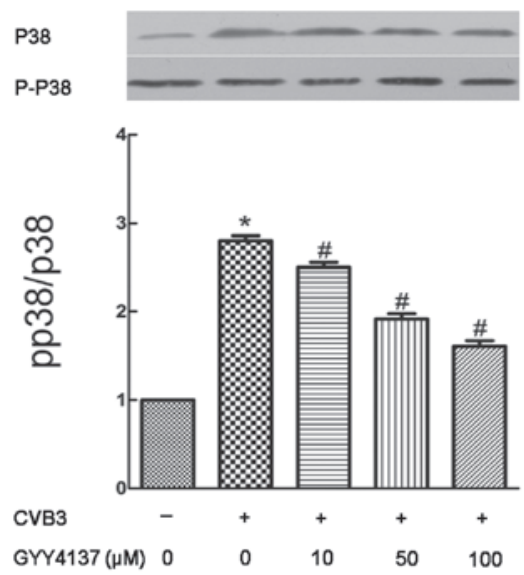

B
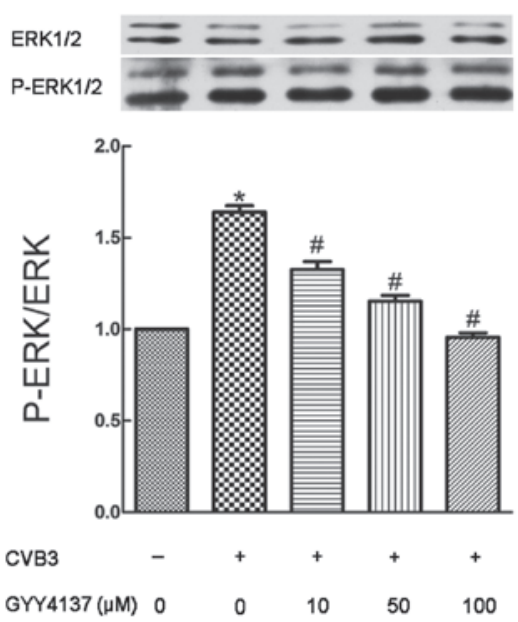

C
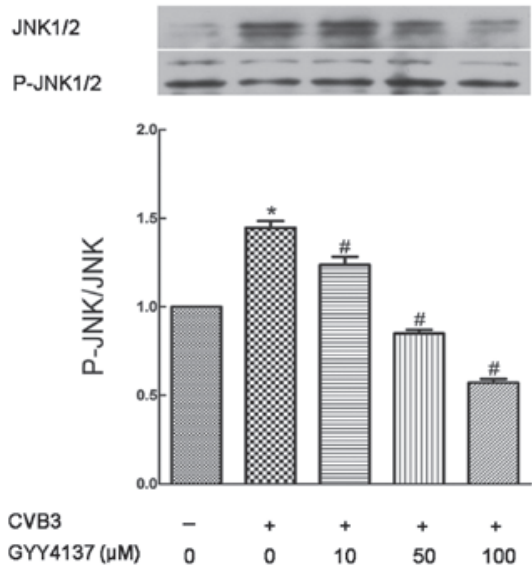

Figure 7. Effects of GYY4137 on mitogen-activated protein kinases in cardiomyocytes infected with Coxsackie virus B3 (CVB3). Following infection with CVB3 for $2 \mathrm{~h}$, the cells were treated with GYY4137 at concentrations of 10,50 and $100 \mu \mathrm{M}$ for another $6 \mathrm{~h}$, with normal cells serving as a control group. The phosphorylation levels of (A) p38, (B) ERK1/2 and (C) JNK1/2 were analyzed by western blotting. The experiments were repeated three times with similar results. ${ }^{*} \mathrm{P}<0.05$ vs. normal control group; ${ }^{\#} \mathrm{P}<0.05$ vs. CVB3 group.

GYY4137 inhibits CVB3-mediated NF- $\kappa B$ DNA binding activity. NF- $\mathrm{\kappa B}$ is a key regulator of numerous genes in all types of cells. The cardiomyocytes were pretreated with 10,50 or $100 \mu \mathrm{M}$ GYY4137 for $12 \mathrm{~h}$ prior to incubation with CVB3 for $2 \mathrm{~h}$. The inhibition of CVB3-induced NF-кB DNA binding 
activity in the cardiomyocytes was detected by EMSA. As shown in Fig. 5, NF- $\kappa$ B DNA binding activity was significantly increased in the CVB3 group, compared with the control group $(\mathrm{P}<0.05)$; however treatment with different GYY4137 concentrations significantly reduced the CVB3-induced NF- $\mathrm{BB}$ DNA binding activity in a dose-dependent manner $(\mathrm{P}<0.05)$. The CVB3-induced DNA binding was significantly reduced by $100 \mu \mathrm{M}$ GYY4137 $(\mathrm{P}<0.05)$.

Effects of GYY4137 on inducing NF- $\kappa B$ and I $\kappa B \alpha$ degradation. To elucidate the possible mechanism of GYY4137-mediated reduction in the secretion of inflammatory cytokines, the $N F-\kappa B$ signaling pathway was evaluated and the effect of GYY4137 treatment on $\mathrm{I} \kappa \mathrm{B} \alpha$ degradation was examined. The data showed that treatment with GYY4137 significantly prevented the I $\kappa \mathrm{B} \alpha$ degradation induced by $\mathrm{CVB} 3(\mathrm{P}<0.05$; Fig. 6A). p65 was expressed mainly in the cytoplasm prior to CVB3 infection (Fig. 6B), but incubation with CVB3 resulted in the accumulation of p65 in the nucleus (Fig. 6C). However, this nuclear translocation of p65 was significantly suppressed by GYY4137 in a concentration-dependent manner $(\mathrm{P}<0.05)$.

Effects of GYY4137 on MAP kinases in CVB3-infected cardiomyocytes. The activation and phosphorylation of the MAPK signaling pathway has been shown to exert a crucial role in CVB3-induced $\mathrm{NF}-\kappa \mathrm{B}$ activation and the subsequent activation of inflammatory mediators (21). To investigate whether MAPK signaling pathway activation is involved in the regulation of inflammatory response by GYY4137 in CVB3-infected cardiomyocytes, MAPK phosphorylation (indicated by the expression levels of phosphorylation (P)-p38, P-ERK1/2 and P-JNK1/2) was analyzed by western blotting. As shown in Fig. 7, P-p38, P-ERK1/2 and P-JNK1/2 expression levels in cells were significantly increased after $2 \mathrm{~h}$ treatment with CVB3 (P<0.05; Fig. 7), but GYY4137 treatment $(10-100 \mu \mathrm{M})$ inhibited this phosphorylation $(\mathrm{P}<0.05)$. These results suggest that GYY4137 may reduce the expression of CVB3-induced inflammatory factors by suppressing the phosphorylation of p38, ERK1/2 and JNK1/2 molecules in the MAPK signaling pathway.

\section{Discussion}

VM is manifested by attacks on infection-induced myocytes. Following viral infection, immune signaling pathways are activated. The presence of Coxsackie virus in host cells and the subsequent immune reaction may result in dilated cardiomyopathy and heart failure (1). Increasing evidence indicates that the myocardial cell inflammatory reaction induced by CVB3 infection is crucial in VM development and myocardial remodeling (2).

The NF- $\kappa$ B signaling pathway is a proinflammatory pathway involved in the regulation of a number of inflammatory responses. A previous study confirmed that the activation of $\mathrm{NF}-\kappa \mathrm{B}$, a nuclear transcription factor is important in the development of CVB3-mediated myocarditis (21). NF- $\kappa \mathrm{B}$ as a central component of the inflammatory reaction regulates the expression levels of various inflammatory factors (21). In CVB3-mediated myocarditis, $\mathrm{NF}-\kappa \mathrm{B}$ is activated and the expression levels of downstream chemical factors, cell adhesion factors and inflammatory factors, such as IL-6, IL-1 $\beta$ and TNF- $\alpha$, markedly increase, thereby further damaging the heart (4). Inactivated $\mathrm{NF}-\kappa \mathrm{B}$ reacts with $\mathrm{I} \kappa \mathrm{B}-\alpha$ to form a compound that is distributed in the cytoplasm. When an inflammatory factor, growth factor or chemokine, is present that activates $N F-\kappa B, I \kappa B-\alpha$ is phosphorylated at Serine 32 and 36 , and then degraded via the ubiquitin-protease pathway. When $\mathrm{NF}-\kappa \mathrm{B}$ and $\mathrm{I} \kappa \mathrm{B}-\alpha$ are depolymerized, the nuclear localization sequences are exposed and the molecules are transported into the nucleolus to combine with the relevant DNA gene sequences, induce the transcription of target genes and accelerate the transcription of $\mathrm{NF}-\kappa \mathrm{B}$-dependent genes, including IL- 6 , IL- $1 \beta$ and TNF- $\alpha$. Therefore, following suppression of the NF- $\mathrm{B}$ pathway, the heart is prevented from undergoing an inflammatory response through the downregulation of proinflammatory factor expression (25).

GYY4137 is a novel $\mathrm{H}_{2} \mathrm{~S}$-releasing agent and has been found to exert anti-inflammatory and anti-apoptotic actions, expand the coronary artery, improve myocardial oxygen supply and protect the heart (22). The anti-inflammatory effect is realized via inhibiting the expression of NF- $\mathrm{B}$-regulated inflammatory genes and proinflammatory factors (23). In the present study, GYY4137 exerted a protective effect, significantly reducing the secretion of IL- 6 , IL-1 $\beta$ and TNF- $\alpha$ proteins following 10-100 $\mu \mathrm{M}$ GYY4137 treatment in myocardial cells subsequent to CVB3 infection. Enzymatic determination of myocardial injury revealed that the levels of LDH and CK-MB were significantly reduced, which alleviated the damage to myocardial cells. This result is concurrent with that of a previous study that reported that $\mathrm{H}_{2} \mathrm{~S}$ pretreatment significantly reduced the inflammatory reaction in a rat total hepatic ischemia and reperfusion model (24).

To analyze the underlying mechanism of how GYY4137 protects CVB3-infected myocardial cells, the effects of GYY4137 on the CVB3-activated NF- $\mathrm{BB}$ signaling pathway were investigated in the present study. The data show that GYY4137 reduced CVB3-mediated $\mathrm{I} \kappa \mathrm{B} \alpha$ degradation in myocardial cells in a concentration-dependent manner, and resulted in $\mathrm{NF}-\kappa \mathrm{B}$ and $\mathrm{I} \kappa \mathrm{B}-\alpha$ depolymerization, thereby resulting in translocation of $\mathrm{NF}-\kappa \mathrm{B}$ from the cytoplasm to the nucleolus. To verify the activation of NF- $\kappa \mathrm{B}$, detection of this change in cellular localisation is important. With the dislocation and activation of $\mathrm{NF}-\kappa \mathrm{B}$, the levels of CVB3-mediated inflammatory factors were significantly increased. Whiteman et al (26) found that GYY4137 restrained the expression of proinflammatory factors in macrophages and upregulated IL-10 expression through negatively affecting $\mathrm{NF}-\kappa \mathrm{B}$ nuclear translocation, thereby inducing an anti-inflammatory reaction (26). To further confirm whether GYY4137 induces an anti-inflammatory reaction through restraining NF- $\kappa$ B DNA binding ability, the effects of GYY413 on this binding ability were examined in the present study. EMSA revealed that CVB3-mediated $\mathrm{NF}-\kappa \mathrm{B}$ DNA binding ability is inhibited in a concentration-dependent manner by GYY4137, indicating that GYY4137 restrains the expression of IL-6, IL-1 $\beta$ and TNF- $\alpha$ through repressing NF- $\kappa$ B DNA binding ability. Therefore, in the CVB3-infected myocardial cells, GYY4137 significantly supressed the expression of these proinflammatory factors by inhibiting the $N F-\kappa B$ signaling pathway. 
The MAPK intracellular signaling pathway is important in inflammatory reactions induced by viral infection and oxidative stress, and is involved in regulating the expression of various inflammatory factors and cell adhesion factors (17). Therefore, to further analyze the underlying molecular mechanism with regard to how GYY4137 inhibits the secretion of proinflammatory factors, the effects of GYY4137 on the MAPK signaling pathway were investigated. A previous study observed that in in vivo and in vitro models of inflammation, the MAPK signaling pathway is involved in the regulation of NF- $\kappa \mathrm{B}$ activation (27). Furthermore, in a mouse VM model, ERK1/2, p38 and JNK1/2 were shown to be activated, and involved in the inflammatory reaction and upregulated expression of inflammatory factors $(28,29)$. In accordance with this, in the present study, $6 \mathrm{~h}$ after CVB3 infection, ERK1/2, p38 and JNK1/2 were significantly activated, as revealed by increased phosphorylation of these molecules. Following intervention with GYY4137, the levels of phosphorylated ERK1/2, p38 and JNK1/2 were inhibited in a concentration-dependent manner. Therefore, GYY4137 is hypothesized to inhibit the activation of the MAPK signaling pathway and thereby suppress the expression of inflammatory factors in CVB3-infected rat myocardial cells.

In conclusion, in the myocardial cells of CVB3-infected rats, GYY4137 downregulated the expression of proinflammatory factors, reduced the levels of myocardial injury-related enzymes (LDH and CK-MB) and alleviated damage to myocardial cells. Thus, GYY4137 may provides a novel therapeutic method in the treatment of VM. The anti-inflammatory effect was achieved by inhibiting the NF- $\kappa \mathrm{B}$ and MAPK signaling pathways. However, whether the anti-inflammatory effect through inhibition of the MAPK signaling pathway is achieved through regulation of $\mathrm{NF}-\kappa \mathrm{B}$ activation requires further investigation.

\section{Acknowledgements}

The authors would like to thank Professor ZhanQiu Yang (State Key Laboratory of Virology) for providing key reagents and technical guidance, and the biotechnicians of the laboratories of Pediatrics and Gastroenterology of Union Hospital (Wuhan, China) for technical support.

\section{References}

1. Esfandiarei M and McManus BM: Molecular biology and pathogenesis of viral myocarditis. Annu Rev Pathol 3: 127-155, 2008.

2. Yajima T and Knowlton KU: Viral myocarditis: from the perspective of the virus. Circulation 119: 2615-2624, 2009.

3. Sobotta K, Wilsky S, Althof N, Wiesener N, Wutzler P and Henke A: Inhibition of nuclear factor kappa B activation reduces Coxsackievirus B3 replication in lymphoid cells. Virus Res 163: 495-502, 2012.

4. Li K, Xu W, Guo Q, et al: Differential macrophage polarization in male and female BALB/c mice infected with coxsackievirus B3 defines susceptibility to viral myocarditis. Circ Res 105: 353-364, 2009.

5. Gui J, Yue Y, Chen R, Xu W and Xiong S: A20 (TNFAIP3) alleviates CVB3-induced myocarditis via inhibiting NF-kappaB signaling. PLoS One 7: e46515, 2012.

6. Predmore BL, Lefer DJ and Gojon G: Hydrogen sulfide in biochemistry and medicine. Antioxid Redox Signal 17: 119-140, 2012.

7. Lowicka E and Beltowski J: Hydrogen sulfide $\left(\mathrm{H}_{2} \mathrm{~S}\right)$ - the third gas of interest for pharmacologists. Pharmacol Rep 59: 4-24, 2007.
8. Bian JS, Yong QC, Pan TT, et al: Role of hydrogen sulfide in the cardioprotection caused by ischemic preconditioning in the rat heart and cardiac myocytes. J Pharmacol Exp Ther 316: 670-678, 2006.

9. Li L, Bhatia M and Moore PK: Hydrogen sulphide - a novel mediator of inflammation? Curr Opin Pharmacol 6: 125-129, 2006.

10. Rinaldi L, Gobbi G, Pambianco M, Micheloni C, Mirandola P and Vitale M: Hydrogen sulfide prevents apoptosis of human PMN via inhibition of p38 and caspase 3. Lab Invest 86: 391-397, 2006.

11. Hu LF, Lu M, Wu ZY, Wong PT and Bian JS: Hydrogen sulfide inhibits rotenone-induced apoptosis via preservation of mitochondrial function. Mol Pharmacol 75: 27-34, 2009.

12. Li L, Bhatia M, Zhu YZ, et al: Hydrogen sulfide is a novel mediator of lipopolysaccharide-induced inflammation in the mouse. FASEB J 19: 1196-1198, 2005.

13. Li T, Zhao B, Wang C, et al: Regulatory effects of hydrogen sulfide on IL-6, IL-8 and IL-10 levels in the plasma and pulmonary tissue of rats with acute lung injury. Exp Biol Med (Maywood) 233: 1081-1087, 2008.

14. Tamizhselvi R, Sun J, Koh YH and Bhatia M: Effect of hydrogen sulfide on the phosphatidylinositol 3-kinase-protein kinase B pathway and on caerulein-induced cytokine production in isolated mouse pancreatic acinar cells. J Pharmacol Exp Ther 329: 1166-1177, 2009.

15. Li L, Salto-Tellez M, Tan CH, Whiteman M and Moore PK: GYY4137, a novel hydrogen sulfide-releasing molecule, protects against endotoxic shock in the rat. Free Radic Biol Med 47: 103-113, 2009.

16. Li L, Fox B, Keeble J, et al: The complex effects of the slow-releasing hydrogen sulfide donor GYY4137 in a model of acute joint inflammation and in human cartilage cells. J Cell Mol Med 17: 365-376, 2013.

17. Hommes DW, Peppelenbosch MP and van Deventer SJ: Mitogen activated protein (MAP) kinase signal transduction pathways and novel anti-inflammatory targets. Gut 52: 144-151, 2003.

18. Zhang H, Moochhala SM and Bhatia M: Endogenous hydrogen sulfide regulates inflammatory response by activating the ERK pathway in polymicrobial sepsis. J Immunol 181: 4320-4331, 2008.

19. Si X, Luo H, Morgan A, et al: Stress-activated protein kinases are involved in coxsackievirus B3 viral progeny release. J Virol 79: 13875-13881, 2005.

20. Zhang C, Lin G, Wan W, et al: Resveratrol, a polyphenol phytoalexin, protects cardiomyocytes against anoxia/reoxygenation injury via the TLR4/NF-kappaB signaling pathway. Int J Mol Med 29: 557-563, 2012.

21. Li Q and Verma IM: NF-kappaB regulation in the immune system. Nat Rev Immunol 2: 725-734, 2002.

22. Szabó C: Hydrogen sulphide and its therapeutic potential. Nat Rev Drug Discov 6: 917-935, 2007.

23. Yang C, Yang Z, Zhang M, et al: Hydrogen sulfide protects against chemical hypoxia-induced cytotoxicity and inflammation in $\mathrm{HaCaT}$ cells through inhibition of ROS/NF-kappaB/COX-2 pathway. PLoS One 6: e21971, 2011.

24. Chen Y, Liu Z and Xie X: Hydrogen sulphide attenuates renal and cardiac injury after total hepatic ischemia and reperfusion. J Surg Res 164: e305-e313, 2010.

25. Hayden MS and Ghosh S: Shared principles in NF-kappaB signaling. Cell 132: 344-362, 2008.

26. Whiteman M, Li L, Rose P, Tan CH, Parkinson DB and Moore PK: The effect of hydrogen sulfide donors on lipopolysaccharide-induced formation of inflammatory mediators in macrophages. Antioxid Redox Signal 12: 1147-1154, 2010.

27. Chen TH, Kao YC, Chen BC, Chen CH, Chan P and Lee HM: Dipyridamole activation of mitogen-activated protein kinase phosphatase-1 mediates inhibition of lipopolysaccharide-induced cyclooxygenase- 2 expression in RAW 264.7 cells. Eur J Pharmacol 541: 138-146, 2006.

28. Yuen S, Smith J, Caruso L, Balan M and Opavsky MA: The coxsackie-adenovirus receptor induces an inflammatory cardiomyopathy independent of viral infection. J Mol Cell Cardiol 50: 826-840, 2011.

29. Marchant D, Dou Y, Luo H, et al: Bosentan enhances viral load via endothelin-1 receptor type-A-mediated p38 mitogen-activated protein kinase activation while improving cardiac function during coxsackievirus-induced myocarditis. Circ Res 104: 813-821, 2009. 